\title{
Effect of nonuniform doping profile on thermometric performance of diode temperature sensors
}

\author{
V.N. Sokolov, Yu.M. Shwarts \\ Institute of Semiconductor Physics, NAS of Ukraine, 45 prospect Nauky, 03028 Kiev, Ukraine
}

\begin{abstract}
A theoretical investigation of the influence of a nonuniform doping concentration to the temperature response curve of diode temperature sensors is presented, which is the first effort in this field important for diffused diodes used as the temperature sensors. The currentvoltage characteristic, from which the temperature response curve can be obtained, has been calculated using the model of a one-dimensional exponentially graded $p-n$ junction with uniformly doped base region and the diffusion current of the minority carriers through the $p-n$ junction. We show that depending on the doping gradient both contributions to the current coming from the electron and hole current components appear to be of the same order of magnitude. That is in contrast to the prediction of the widely used asymmetrical step junction model. It follows from numerical calculations that an effective shift of the temperature response curve due to nonuniformly doped emitter region in the temperature equivalent can reach the value of about $20 \mathrm{~K}$. The limiting temperature $\mathrm{T}_{\mathrm{m}}$ in the temperature response curve that restricts its extent into the high temperature range has been analyzed depending on the excitation current, the doping concentration of the base, and the $p-n$ junction depth.
\end{abstract}

Keywords: silicon diode structure, temperature sensor, temperature response curve, graded junction.

Paper received 27.02.02; revised manuscript received 19.04.02; accepted for publication 25.06.02.

\section{Introduction}

The development of wide-range, high-precision and reproducible diode temperature sensors (DTSs) based on a $p-n$ junction, even using the most advanced in semiconductor engineering silicon technology, is not a simple scientific and technological problem. From the viewpoint of basic physics of the development of silicon devices with a $p-n$ junction, with well-predicted and reproducible characteristics, an optimal carrier transport mechanism in the diode structure is the diffusion current of the minority carriers [1]. In our previous studies, we have shown that the measured temperature range, the temperature response curve and sensitivity of such optimized DTSs are determined by a set of the electrophysical (impurity concentration, minority carrier lifetime and diffusion length, excitation current value of the sensor) and design $(p-n$ junction area, the base length of a diode) parameters of $p-n$ junction, interrelated by the self-consistent optimi- zation method of the sensor [2,3]. It is to be noted that DTSs are applied as precision temperature measuring devices, often operating in limiting regimes with measurement accuracy of the order of hundredth parts of degree. Realization of high measurement accuracy, as well as physical approximation of thermometric characteristics and improvement of interchangeability of DTSs, demand the most complete taking account of the role of main parameters of diode structures for theoretical description of the carrier transport through $p-n$ junction.

Current-voltage characteristics (CVCs) of real diodes used for the development of DTSs demonstrate the ideality factor value $m>1$ [4], which is indicative of considerable contribution to the electric transport not only due to the diffusion current but also currents of different origin (the generation-recombination current [5-7], the tunneling current [8-10], and others [7,11]). Silicon $p-n$ junctions with nearly ideal CVCs are mentioned in the literature as a technological achievement in the field of development of semiconductor devices with predicted and reproduc- 


\section{V.N. Sokolov et al.: Effect of nonuniform doping profile...}

ible characteristics [7]. The diffusion technology developed by us (a sequence of technological operations in combination with the established gettering and soft thermal treatment regimes) provides making the diodes with CVCs close to the ideal in a rather wide range of temperatures $(130 \div 500 \mathrm{~K})$ and currents $\left(10^{-8} \div 10^{-4} \mathrm{~A}\right)[12]$. Since in the diffused $p-n$ junctions there always exists a nonuniform doping profile (both in the emitter and base regions), it influences the $p-n$ junction parameters [13] and the minority carrier transport [14,1]. The carrier transport peculiarities in nonuniformly doped structures with $p-n$ junction are used, for instance, in drift transistors $[15,1]$ and semiconductor solar sells [16].

Until the present, the calculations of thermometric characteristics of DTSs for most widespread semiconductor materials (Ge, $\mathrm{Si}, \mathrm{GaAs})$ are based on the simplest model of a step $p-n$ junction [17-19]. In this approach, the following important factors are ignored such as the occurrence of built-in (diffusion) electric field and distribution of the effective lifetime of nonequilibrium carriers in the emitter and base regions of the diode associated with nonuniform doping profile. Taking into account these factors in the theory results in a radical change of corresponding expression for the diode CVC in comparison with the ideal CVC [1], which is essential for determination of the temperature response curve of DTSs. This paper presents a first theoretical analysis and corresponding calculations of the influence of nonuniform doping profile on the temperature response curve of DTS with predominant diffusion minority carrier transport. Taking into account the above mentioned factors essential for diffusion technology of manufacturing the thermodiode sensors, these theoretical calculations not only make more exact notions about nonequilibrium processes in the $p-n$ junction, but also allow to supplement a view of physical mechanisms responsible for the formation of the DTS characteristics.

\section{Qualitative analysis}

The temperature response curve (TRC) is one of the basic characteristics of DTS and determined by a temperature $T$ dependence of the voltage drop $U=U(T, I)$ across the diode at a given forward current $I$, and the sensitivity $\alpha(T, I)=\partial U(T, I) / \partial T$. The TRC of a sensor can be calculated theoretically resulting from the known expression for CVC $I=I\left(U, T ; A_{i}\right)$ of the diode at a given temperature (coefficients $A_{i}$ stand for a set of electrophysical parameters determining the carrier transport).

To calculate CVC, definite model notions are required. The ideal CVC (1) is derived using the following assumptions [1]: 1) Approximation of depletion layer (DL) with abrupt boundaries with electroneutral regions of emitter and base having negligibly small resistance in comparison with that of the DL; 2) Boltzmann statistics for distrtributions of electrons and holes on the energy at full ionization of doping impurities; 3) Low level of injection; 4) Absence of the generation-recombination current in the DL. In so doing, it is highly essential that the DL approximation is constructed for a stepwise (one-dimensional) distribution of doping impurities. Then the CVC is described by the Shockly formula [1]

$I=S j_{S}\left(T, A_{i}\right)\left(e^{q U / k_{B} T}-1\right)$,

where $S$ is an effective area of the junction, $j_{s}=j_{s n}+j_{s p}$ the saturation current density, $q$ absolute value of the electron charge, $k_{B}$ the Boltzmann constant. The ratio between the hole $j_{s p}$ and electron $j_{s n}$ components of the current (1) is $[6,20]$

$\frac{j_{s p}}{j_{s n}}=\frac{N_{A}}{N_{D}}\left(\frac{D_{p} \tau_{n}}{D_{n} \tau_{p}}\right)^{1 / 2} \frac{\operatorname{th}\left(d_{p} / L_{n}\right)}{\operatorname{th}\left(d_{n} / L_{p}\right)}$,

where $N_{D, A}$ is the concentration of donors or acceptors, $D_{n, p}$ and $\tau_{n, p}$ the diffusion coefficient and the lifetime for electrons $(n)$ and holes $(p)$, respectively, $L_{n, p}=$ $\left(D_{n, p} \tau_{n, p}\right)^{1 / 2}$ the characteristic diffusion lengths, $d_{n, p}$ the length of the $n$-and $p$-regions of the diode.

In the case of asymmetrical $p-n$ junction considered below for definitness of the model (Fig. 1), the main contribution to the current (1) comes from the holes injected by $p-n$ junction and diffusing within the $\mathrm{n}$-base of the diode towards the $n^{+}$-contact: $j_{s} \approx j_{s p}$. The contribution of analogous component of the diffusion current of the electrons $j_{s n}$ will be small, since for an asymmetrical junction a strong inequality is fulfilled: $\left(j_{s n} / j_{s p}\right) \approx\left(N_{D} / N_{A}\right)<<1$. As a result, the contribution of $j_{s n}$ in the expression for $\mathrm{CVC}$ (1), with a good accuracy, may not be taken into consideration. For instance, for the sensors [21], in which diode $n^{+}-p$ structures are used as a sensitive element, the ratio of the acceptor concentration $\left(\sim 2 \cdot 10^{17} \mathrm{~cm}^{-3}\right)$ to the donor one $\left(\sim 10^{20} \mathrm{~cm}^{-3}\right)$ constitutes $0.2 \%$. The contribution of the diffusion current of electrons to the TRC of the sensor will be yet less considerable, since the TRC corresponding to the CVC (1) is changed on a logarithmic law with the change of the $j_{s}$ value:

$$
U(T, I)=\frac{k_{B} T}{q} \ln \left[\frac{I}{S j_{S}\left(T, A_{i}\right)}+1\right] .
$$

For asymmetrical junctions that are applied in diffused silicon diode structures as the sensitive elements of DTSs, the gradient of impurity concentration in the heavily doped emitter region of the diode considerably exceeds that in the base. The magnitude of built-in (diffusion) electric field in the emitter $E_{p r}$ can reach the values compared with the electric field strength in the DL of $p$ $n$ junction. The occurrence of the field which distribution is not restricted by an immediate vicinity of metallurgical boundary of the junction, as it takes place for a stepwise impurity profile, as well as high concentration of free (majority) carriers result in characteristic features 
V.N. Sokolov et al.: Effect of nonuniform doping profile...

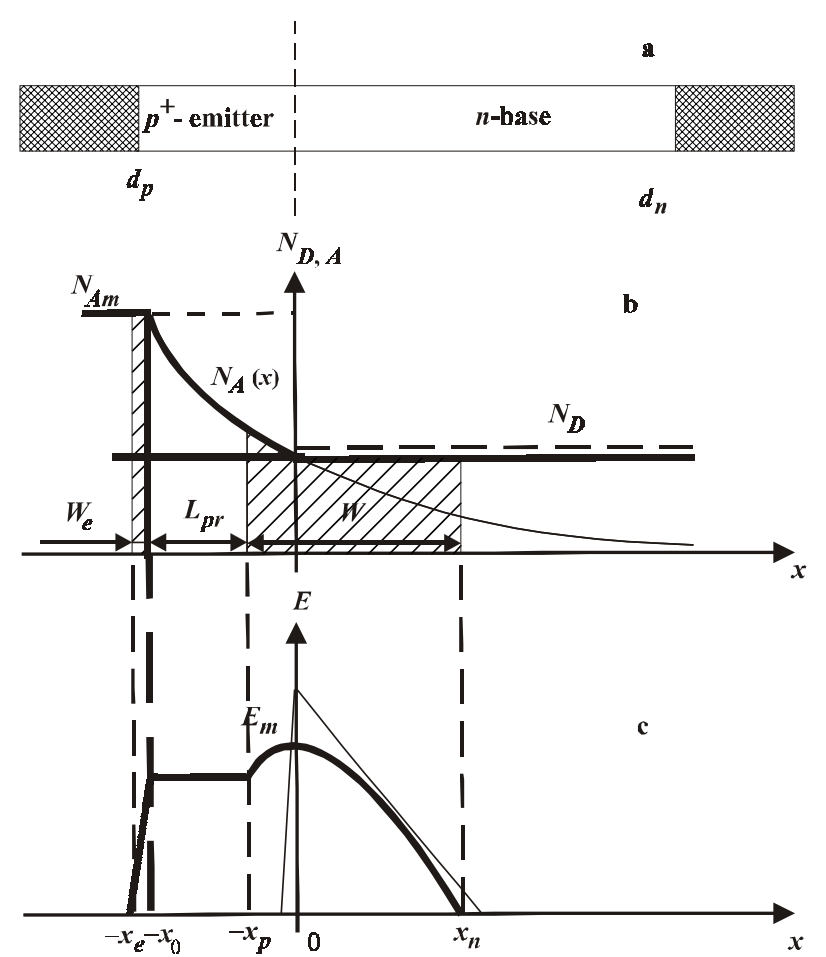

Fig. 1. Schematic view of a diode based on the diffused $p-n$ junction (dashed area indicates ohmic contacts) (a); spacecharge region (dashed) and distribution of donor $N_{D}$ and acceptor $N_{A}$ (b) concentrations; electric field distribution (c). Dashed lines - stepwise impurity distribution model, solid lines - exponential model. $N_{A m}$ is maximum acceptor concentration in the emitter, $x_{0}$ is the depth of metallurgical boundary $(x=0)$ of the $p-n$ junction, $x_{n, p}$ are the DL boundaries of the $p-n$ junction, $E_{m}$ is the maximal field, $x_{e}, 0$ are the boudaries of DL near the emitter contact, $d_{n}$ and $d_{p}$ are the extent of the $n-$ and p-region of the diode.

of structure of the space-charge region [22]. Indeed, since the Debye screening length in the emitter $l_{D p}$ is rather small $\left(l_{D p}<x_{0}\right)$, for its main part $\left(\approx x_{0}\right)$ the quasineutrality condition is valid: $\rho(x)=0, E_{p r}(x)=$ const, where $\rho(x)$ is the charge density. Thus, rather extended region of approximately constant (built-in) field is an intermediate one between the DL of $\mathrm{p}-\mathrm{n}$ junction and the surface DL at the $p^{+}$-contact, where outlet is provided for the electrostatic potential to a constant value.

Taking account of the nonuniform doping profile in an adequate model of the junction results in not only characteristic features in distributions of the charge, potential, and electric field but also affects substantially the electric transport. The boundary conditions on the concentration of minority carriers used for specific calculation of CVC are modified; the occurrence of the field $E_{p r}$ results in the need to take into account the drift component of the minority carrier current, characteristic lifetime of which in the region with nonuniform distribution of impurities is position dependent. Besides, since the impurity concentration at the edge of DL of $p-n$ junction on the emitter side is considerably less than that at the contact, there arises an effective symmetrization (a loss of strong asymmetry) of the junction, and there increases the efficiency of injection of the minority carriers into the emitter region. In the case, both components of the minority-carrier current (electrons and holes) become comparable in its magnitude. The dependence of this boundary location relatively the impurity concentration profile on applied voltage $U$, as well as the set of all above mentioned factors, result in that saturation current density in corresponding formula for $\mathrm{CVC}$ becoming dependent on $U$. Below, an analytical calculation of TRC of diode temperature sensor is adduced on the basis of model of exponential doping profile in $p-n$ junction using the assumptions 1) - 4), which, in its results, differs on principle from the model with stepwise distribution of impurities.

\section{Basic equations}

To calculate CVC of the diode in the accepted approximation, it is necessary to compute the diffusion current density of minority carriers at the boundaries of DL of the $p-n$ junction with quasi-neutral regions of the diode. In turn, it is necessary for that to find distributions of minority carriers injected by the $p-n$ junction in the emitter and base. Such a problem corresponds to solving a system of the second-order differential equations - continuity equations for the concentration of electrons $n$ and holes $p$ and Poisson's equation for the potential $\varphi$ of electric field $E=-\nabla \varphi$ :

$$
\begin{aligned}
& \frac{\partial n}{\partial t}-\frac{1}{q} \operatorname{div} \mathrm{j}_{n}=G_{n}-R_{n} \\
& \frac{\partial p}{\partial t}+\frac{1}{q} \operatorname{div} \mathrm{j}_{p}=G_{p}-R_{p} \\
& \Delta \varphi=4 \pi \rho
\end{aligned}
$$

where $G_{n, p}$ and $R_{n, p}$ are the carrier generation and recombination rate functions. The system (4) - (6) is supplemented by boundary conditions at the edges of DL and contacts as well as by expressions for the electron and hole components of the current density

$j_{n}=q \mu_{n} n \mathrm{E}+q D_{n} \nabla n, j_{p}=q \mu_{p} p \mathrm{E}-q D_{p} \nabla p$,

$\mu_{n, p}$ is the electron and hole mobility. For $n-$ and $p$-region of the diode outside the DL, we use instead of (6) the equation of quasineutrality

$\Delta n \equiv n-n_{0}=p-p_{0} \equiv \Delta p$,

$n_{0}$ and $p_{0}$ are equilibrium values of the concentrations $n$ and $p$. The validity criterion for (8) is smallness of the Debye screening lengths $l_{D n}$ and $l_{D p}$ in those regions in comparison with other characteristic lengths of the problem (characteristic length scale of impurity distribution, diffusion length of electrons $L_{n}$ and holes $L_{p}$ ). 
Further for equations (4) - (6) the conditions are accepted of stationarity $[(\partial n / \partial t)=(\partial p / \partial t)=0]$,onedimensionality (dependence of the variables on coordinate $x)$, as well as the absence of generation $\left(G_{n}=G_{p}=0\right)$ and equality of the electron and hole lifetimes in each of quasi-neutral regions $\left(R_{n}=R_{p}\right)$. Instead of equations (4), (5), it is convenient to use their combination formed as follows. Subtracting from equation (5) equation (4), we obtain $d j / d x=0$. From here it follows that the current density $j$ must be constant

$$
j=j_{n}+j_{p}=\text { const },
$$

Substituting the expressions for $j_{n}$ and $j_{p}$ from (7) into (9), we find the electric field distribution

$E(x)=\frac{\frac{1}{q} j+D_{p} \frac{d p}{d x}-D_{n} \frac{d n}{d x}}{n \mu_{n}+p \mu_{p}}$.

Multiplying (4) with $p \mu_{p}$ and (5) with $n \mu_{n}$ and adding both equations, we obtain an equation typical for bipolar drift-diffusion minority-carrier transport

$$
\begin{aligned}
& \frac{D}{n+p}\left[\frac{p}{D_{n}} \frac{d}{d x}\left(D_{n} \frac{d n}{d x}\right)+\frac{n}{D_{p}} \frac{d}{d x}\left(D_{p} \frac{d p}{d x}\right)\right]+ \\
& +\frac{\mu}{p-n} E\left[\frac{p}{\mu_{n}} \frac{d}{d x}\left(\mu_{n} n\right)-\frac{n}{\mu_{p}} \frac{d}{d x}\left(\mu_{p} p\right)\right]-\frac{n-n_{0}}{\tau}=0,
\end{aligned}
$$

with

$$
D=\frac{n+p}{\left(n / D_{p}\right)+\left(p / D_{n}\right)}, \quad \mu=\frac{p-n}{\left(n / \mu_{p}\right)+\left(p / \mu_{n}\right)}
$$

being the bipolar diffusion coefficient and drift mobility, respectively. Here we have used the Einstein relation $\mu_{n, p}=q D_{n, p} / k_{B} T$. Solving equations (11), (8) together with $(10),(12)$, one can find distribution of concentrations of the minority carriers - holes in the $n$-region $\left(\tau=\tau_{\mathrm{p}}\right)$ and electrons in the $p$-region $\left(\tau=\tau_{n}\right)$. A peculiarity of this equation distinguishing it from equations (4) and (5) is the absence of term with gradient of the electric field, which simplifies its structure. Specific form of distribution of concentrations $n(x)$ and $p(x)$ depends on the nonuniform doping profile, as well as on the respective boundary conditions. For a stepwise (quasi-uniform) impurity distribution, (11) reduces into the standard equation for bipolar diffusion in a homogeneous sample [1].

\section{Distribution of the minority carriers in quasi- neutral regions of the diode}

\section{A. Model of impurity distribution}

Let us consider the heavily doped $p+-$ region (Fig. 1). The nonuniform distribution of main impurities (acceptors) is approximated by an exponential function

$N_{A}(x)=N_{A m} e^{-\left(x+x_{0}\right) / \lambda}$,

where $\lambda=x_{0} / \eta$ is characteristic length scale of changing the doping impurity concentration, $x_{0}=d_{p}$ is the depth of metallurgical boundary $(x=0)$ of the $p-n$ junction, $N_{A m}=N_{D} \exp \left(x_{0} / l\right)$ is the maximum acceptor concentration. The parameter $\eta=\ln \left(N_{\mathrm{A} m} / N_{D}\right)$ characterizes the degree of asymmetry of the diode doping [in the model of stepwise impurity distribution for a fully symmetrical junction $\left(N_{D}=N_{A}\right)$ the parameter $\eta$ equals to zero]. The distribution of the donor concentration $N_{D}$ is assumed to be uniform. The exponential model (13) allows one to carry out calculations of characteristics of semiconductor devices based on $p-n$ junction in an analytical form, which is convenient for a further analysis and optimization [15,22-24].

Solving the nonlinear differential equation (11) in general case of an arbitrary impurity profile is possible only with the aid of numerical methods, which highly complicates an analysis of the considered situation. For the model of exponentially graded $p-n$ junction, the coefficients at derivatives in (11) are reduced to constants and the general solution is succeeded to write in an analytical form. For an asymmetrical junction $\left(N_{A m}>>N_{D}\right)$ and for low level of injection from (12) it follows $D \approx D_{\mathrm{n}}$, $\mu \approx \mu_{n}$. In the graded region the mobility and diffusivity may be position dependent, since the carrier mean free path changes with the change of impurity concentration if the carrier scattering on impurities contributes remarkably to transport coefficients. Here, such a dependence is assumed to be nonessential for TRC in the form of (3). Correspondingly, the magnitude of $D_{n}$ and $\mu_{n}$ is taken to be constant. Besides, in (10) one may neglect the field component associated with ohmic voltage drop across this region (Introduction, item 1) as well as to consider that the electric field distribution is mainly formed by gradient of the majority carriers (Introduction, item 3). Therefore, one may write approximately for the field $E=E_{p r}$, where

$$
E_{p r}=\frac{D_{p}}{\mu_{p}} \cdot \frac{1}{p_{0}} \frac{d p_{0}}{d x}=-\frac{k_{B} T}{q \lambda} .
$$

The corresponding criterion is determined by the set of inequalities

$$
\frac{D_{n} n_{i}^{2}}{D_{p} p_{0}^{2}\left(-x_{p}\right)} \ll<1, \quad \frac{D_{n} \lambda \Delta n\left(-x_{p}\right)}{D_{p} L p_{0}\left(-x_{p}\right)} \ll<1, \quad \frac{I}{I_{0}} \ll<,
$$


V.N. Sokolov et al.: Effect of nonuniform doping profile...

where $I_{0}=S q D_{p} N_{D} x_{0} / \lambda^{2}$ is a characteristic current, and the length $L$ is a scale of nonuniform distribution of the nonequlibrium electrons. The left-hand side of these inequalities represent relative contribution into the field (10), respectively, of the diffusion field arising due to nonuniform distribution of electron concentration and ohmic voltage drop across the considered region with the resistance $R=\lambda /\left[q \mu_{p} p_{0}\left(-x_{p}\right) S\right]$, where for a forward bias diode one may take $p_{0}\left(-x_{p}\right) \cong N_{D}$. As a result, equation (11) for the minority carrier concentration (electrons) in the quasi-neutral $p^{+}$-region takes the form

$\frac{d^{2} \Delta n}{d x^{2}}-\frac{1}{\lambda} \frac{d \Delta n}{d x}-\frac{\Delta n}{L_{n}^{2}(x)}=0$,

where $L_{n}(x)=\left[D_{n} \tau_{n}(x)\right]^{1 / 2}$.

\section{B. Recombination model}

The coordinate dependence of the lifetime of nonequilibrium holes is taken into consideration using the Shockley-Read-Hall recombination model through local centers. It is assumed that the centers are filled with holes and injection of electrons is accompanied by their capture into these centers and recombination. Due to a high concentration of holes in the emitter region and low level of injection such capture should not influence considerably the degree of center filling. In this case, one may take that the coordinate dependence of the lifetime $\tau_{n}(x)$ follows the spatial distribution of recombination centers $N_{R}(x)$. For the latter we suppose that it corresponds to distribution (13) of the major impurity concentration

$$
N_{R}(x)=N_{R m} e^{-\left(x+x_{0}\right) / \lambda},
$$

i.e., it has the same scale $\lambda$. At that the bulk lifetime of nonequilibrium electrons for the $p^{+}$-region is described by the dependence

$$
\tau_{n}(x)=\tau_{n 0} e^{x / \lambda},
$$

where $\tau_{n 0} \equiv \tau_{n}(0)$. The minimum lifetime $\tau_{n m} \equiv \tau_{n}\left(-x_{0}\right)=$ $=1 /\left(\gamma_{n} N_{R m}\right)$ corresponds to the maximum concentration of recombination centers $N_{R m}$ in the $p^{+}$-region; where $\gamma_{n}$ is the electron capture coefficient.

\section{Boundary conditions}

The boundary conditions to equation (15) are set at the boundaries of the considered region with the $p^{+}$-contact at $x=-x_{0}$ and with the DL of $p-n$ junction at $x=-x_{p}$ (Fig. 1). The boundary condition at the $p^{+}$-contact has the form [1]: $j_{n}\left(-x_{0}\right)=q s \Delta n\left(-x_{0}\right)$, i.e., the electron current density on this boundary is determined by the density of surface recombination flow characterized by the velocity $s$. With the expression for $j_{n}(7)$ taken into account, we have the boundary condition in the form
$\left.D_{n} \frac{d \Delta n}{d x}\right|_{x=-x_{0}}+\mu_{n} E_{p r} \Delta n\left(-x_{0}\right)=s \Delta n\left(-x_{0}\right)$.

In the limiting cases corresponding to the infinite and zero surface recombination velocity, the following relations should be valid at this boundary:

$\Delta n\left(-x_{0}\right)=0, s=\infty$,

$\left.\frac{d \Delta n}{d x}\right|_{x=-x_{0}}=\frac{1}{\lambda} \Delta n\left(-x_{0}\right), \quad s=0$.

The boundary condition at $x=-x_{p}$ formally has the same form as for a stepwise impurity distribution [1]

$\Delta n\left(-x_{p}\right)=n_{0}\left(-x_{p}\right)\left(e^{q U / k_{B} T}-1\right)$,

where $U$ is the voltage drop directly on the DL of $p-n$ junction.

\section{Solution of equation for minority carrier concentration}

Instead of unknown function $\Delta n(x)$, let us consider a new function $y(z)$, being determined by parametrical dependences

$$
y(x)=e^{-x / 2 \lambda} \Delta n(x), \quad z(x)=\frac{2 \lambda}{L_{n}(x)} .
$$

Equation (15) by substitution (21) is reduced to the Bessel equation

$$
z^{2} \frac{d^{2} y}{d z^{2}}+z \frac{d y}{d z}-\left(z^{2}+v\right) y=0
$$

of the order of $v=1$. The general solution for $v=1$ takes the form [25]

$$
y(z)=C_{1} I_{1}(z)+C_{2} K_{1}(z),
$$

where $I_{l}(z)$ and $K_{l}(z)$ are modified Bessel functions of the first and second orders (McDonald's function of the order of $v=1$ ), respectively; $C_{1,2}$ are the integration constants.

The nonequilibrium electron distribution in the region with the ohmic $p^{+}-$contact $(s=\infty)$ is obtained by utilizing the boundary conditions (19a) and (20). Using the boundary conditions, we find the integration constants

$$
C_{1}=-\Delta n\left(-x_{p}\right) \frac{K_{1}\left(z_{o}\right)}{\Delta\left(z_{p}\right)} e^{x_{p} / 2 \lambda},
$$


$C_{2}=\Delta n\left(-x_{p}\right) \frac{I_{1}\left(z_{0}\right)}{\Delta\left(z_{p}\right)} e^{x_{p} / 2 \lambda}$.

Here, we have introduced a function

$\Delta(z)=I_{1}\left(z_{0}\right) K_{1}(z)-I_{1}(z) K_{1}\left(z_{0}\right)$

the parameters $z_{0} \equiv z\left(-x_{0}\right)$ and $z_{p} \equiv z\left(-x_{p}\right)$ in the argument of the Bessel functions are given by expressions: $z_{0, p}=2 \lambda / L_{n}\left(-x_{0, p}\right)$. Substituting the constants $C_{1,2}$ from (24) into the general solution (23), we find distribution of the excess electron concentration in the $p$-region of the diode

$$
\Delta n(x)=\Delta n\left(-x_{p}\right) \frac{\Delta(z)}{\Delta\left(z_{p}\right)} e^{\left(x+x_{p}\right) / 2 \lambda} .
$$

Similarly, one may obtain distribution of the concentration $\Delta n(x)$ for $s=0$, as well as in the general case of an arbitrary magnitude of the surface velocity. For that, it is necessary to use the boundary conditions (19b), or of the general form (18). Distribution of the nonequilibrium hole concentration in the base is determined by an equation analogous to (11), in which, however, the drift term will be less in the ratio $\lambda_{r}=\lambda_{A} / \lambda_{D}\left(\lambda_{r}<<1\right)$. A specific magnitude of $\lambda_{r}$ depends on characteristic scales of acceptor distribution in the emitter $\lambda_{A}$ and donor distribution in the base $\lambda_{D}$. Below we take the minority carrier distribution (holes) in the base to have a standard form [1] inherent to the diffusion carrier transport at uniform doping.

\section{Current-voltage characteristic}

The current density through the diode, in accordance with assumptions of the model accepted (Introduction, items 1 and 4 ) is determined by a sum of its electron $j_{n}$ and hole $j_{p}$ components calculated at the boundaries of the DL of $p$ $n$ junction [1]: $j=j_{n}\left(-x_{p}\right)+j_{p}\left(x_{n}\right)$ where

$$
\begin{aligned}
& j_{p}\left(x_{n}\right)=-\left.q D_{p} \frac{d p}{d x}\right|_{x=x_{n}}, \\
& j_{n}\left(-x_{p}\right)=q \mu_{n} \operatorname{En}\left(-x_{p}\right)+\left.q D_{n} \frac{d n}{d x}\right|_{x=-x_{p}} .
\end{aligned}
$$

Formulae (26), (27) allow to compute CVC of the diode with nonuniform doping profile. By using expression (14) for the electric field in the quasineutral $p$-region and the Einstein relationship for the connection of the mobility $\mu_{n}$ with the diffusion coefficient $D_{n}$, we rewrite (27) as

$$
j_{n}\left(-x_{p}\right)=\frac{q D_{n} \Delta n\left(-x_{p}\right)}{\lambda}\left(\left.\frac{\lambda}{\Delta n} \frac{d \Delta n}{d x}\right|_{x=-x_{p}}-1\right) .
$$

Using (25) and recurrence relationships for the Bessel functions [25], relating the derivatives of the functions $I_{1}(z)$ and $K_{1}(z)$ with the functions $I_{0,1}(z)$ and $K_{0,1}(z)$, we find expression for the electron current density

$$
j_{n}\left(-x_{p}\right)=\frac{q D_{n} \Delta n\left(-x_{p}\right)}{L_{n}\left(-x_{p}\right) F\left(z_{o}, z_{p}\right)},
$$

in which a function $F\left(z_{0}, z_{p}\right)$ is defined by

$$
F\left(z_{0}, z_{p}\right)=\frac{\Delta\left(z_{p}\right)}{I_{1}\left(z_{0}\right) K_{0}\left(z_{p}\right)+I_{0}\left(z_{p}\right) K_{1}\left(z_{0}\right)} .
$$

Thus, the current-voltage dependence for $p^{+}-n$ structure with nonuniform doping profile in the emitter region of the diode, on the basis of formulae (26) and (29), has the form

$j=j_{S}(T, U)\left(e^{q U / k_{B} T}-1\right)$,

where $j_{s}(T, U)=j_{s n}(T, U)+j_{s p}(T)$ is the saturation current density. The expression for $j_{s p}(T)$, corresponding to the diffusion of holes in the base, has the same form as for a stepwise impurity distribution model:

$j_{s p}(T)=\frac{q D_{p} n_{i}^{2}}{N_{D} L_{p} t h\left(d_{n} / L_{p}\right)}$.

The electron component $j_{s n}(T, U)$, corresponding to the diffusion and drift of electrons in the emitter region of the diode, depends on voltage $U$ :

$$
j_{s n}(T, U)=\frac{q D_{n} n_{i}^{2}}{N_{A}\left(-x_{p}\right) L_{n}\left(-x_{p}\right) F\left(z_{0}, z_{p}\right)} .
$$

Such a dependence is determined by that the concentration $N_{A}$ and the lifetime $\tau_{n}$ are local quantities distributed in the emitter, and it is realized in (33) via the dependence on voltage of the DL boundary $x_{p}=x_{p}(U)$, as well as due to the drift of electrons in the electric field (14).

For not very large magnitudes of voltage $U$ (corresponding to low injection level, however, such that $\left.\exp \left(q U / k_{B} T\right)>>1\right) \mathrm{CVC}(31)$ is characterized for forward branch by a generalized ideality factor $m=\left(q / k_{B} T\right)[d U / d(\operatorname{lnj})]$ close to unity,

$$
m=\frac{1}{1+\frac{k_{B} T}{q} \frac{d}{d U}\left[\ln j_{S}(T, U)\right]}
$$

and for reverse branch $j \approx j_{S}(T, U)$. Here, a distinction of $m$ from unity is caused by the dependence of the preexponential factor $j_{s}(T, U)$ on $U$ in the formula (31) that arises for a nonuniform impurity distribution and is absent for the model of a stepwise impurity distribution in the diode.

Now let us consider how the formula (33) for the electron component of the saturation current density is modi- 


\section{V.N. Sokolov et al.: Effect of nonuniform doping profile...}

fied, if the minority carrier lifetime in the emitter $\tau_{\mathrm{n}}$ does not depend on the coordinate $x$. In this case (15) converts into equation with constant coefficients, solution of which is written by the standard method. Spatial distribution of minority carriers in the emitter will have the form (25) with the change $\Delta(z) \rightarrow \Delta(x)$ и $\Delta\left(z_{p}\right) \rightarrow \Delta\left(-x_{p}\right)$ where

$\Delta(x)=-2 \operatorname{sh}\left[k_{0}\left(x_{0}+x\right) / L_{n}\right], k_{0}=\sqrt{1+L_{n}^{2} /(2 \lambda)^{2}}$.

In (33) one has to make the respective change: $L_{\mathrm{n}}\left(-x_{p}\right) \rightarrow$ $L_{n}=\left(\mathrm{D}_{n} \tau_{n}\right)^{1 / 2}$ and $F\left(z_{0}, z_{p}\right) \rightarrow F\left(x_{0}, x_{p}\right)$, where

$$
F\left(x_{0}, x_{p}\right)=\frac{1}{k_{0} \operatorname{cth}\left[k_{0}\left(x_{0}-x_{p}\right) / L_{n}\right]-L_{n} /(2 \lambda)} \text {. }
$$

The characteristic length $L$ of nonuniform distribution of the minority carriers in the emitter region is determined by a compressed diffusion length (due to the field $\left.E_{p r}\right)$ for which, in view of (25), one can write approximately $L=L_{n} /\left[k_{0}+L_{n} /(2 \lambda)\right]$. In particular, in the limiting case $L_{n} / \lambda<<1$, the influence of electric field $E_{p r}$ on the minority carrier transport can be neglected. Hence, we have $k_{0}=1, L_{\mathrm{ch}}=L_{n}$, and the function $F\left(x_{0}, x_{p}\right)=$ $=\operatorname{th}\left[\left(x_{0}-x_{p}\right) / L_{n}\right]$. Therefore, the saturation current density (33) will have the form, analogous to the formula (32) for stepwise impurity distribution. In the opposite limiting case $L_{n} / \lambda>>1$, we obtain, respectively $k_{0}=L_{n} /(2 \lambda)$, $L_{\mathrm{ch}}=\lambda$, and the function $F\left(x_{0}, x_{p}\right)=2 \lambda\left\{L_{n}\left[\operatorname{cth}\left(x_{0}-x_{p}\right) / 2 \lambda-1\right]\right\}$. For the saturation current density (33), we obtain the expression

$$
j_{s n}(T, U)=\frac{q D_{p} n_{i}^{2}}{2 \lambda N_{A}\left(-x_{p}\right)}\left\{\operatorname{cth} \frac{x_{0}-x_{p}}{2 \lambda}-1\right\} .
$$

\section{Temperature response curve}

As seen from CVC (31), the expression for TRC of sensor based on the diode with nonuniform doping profile cannot be written an explicit form, in contrast to the model of stepwise impurity distribution (3). The dependence $U=U(T, I)$ for a given current value $I$ can be obtained by numerical solution of equation (31) or equivalent equation analogous to (3) in which the saturation current is determined by formulae (32) and (33), with the parameters $x_{n}(U)$ and $x_{p}(U)$ being solutions of transcendental equation system (П5) given in Appendix.

An analysis of the ratio expression between electron (33) and hole (32) current components

$$
\frac{j_{s n}}{j_{s p}}=\frac{N_{D} D_{n} L_{p} \operatorname{th}\left(d_{n} / L_{p}\right)}{N_{A}\left(-x_{p}\right) D_{p} L_{n}\left(-x_{p}\right) F\left(z_{0}, z_{p}\right)}
$$

allows to make the following conclusion concerning the influence of impurity doping profile to CVC and TRC of the diode temperature sensor:
1. Impurity distribution with a finite length scale $\lambda$ results in effective symmetrization of $p-n$ junction, since it appears that $\left(N_{D} / N_{A m}\right)<<\left[N_{D} / N_{A}\left(-x_{\mathrm{p}}\right)\right]<1$. By that reason, electron component of the drift-diffusion current in the emitter can reach values compared with hole component of the diffusion current in the base.

2. CVC of the diode with nonuniform doping profile shifts towards an increasing current density (for a given value of voltage) in comparison with CVC of a step $p-n$ junction.

3. TRC of the diode sensor shifts towards less voltage magnitudes, and its extent to the range of high temperatures decreases, respectively the sensitivity increases. An analogous shift of TRC takes place in the case of decreasing asymmetry degree for a stepwise $p-n$ junction with the change of doping level $[2,3]$.

4. The dependence of CVC and TRC on the emitter region length, arising under conditions of nonuniform doping, is determined by a concurrence between the following factors: diffusion spreading of the minority carriers; influence of the built-in electric field on the minority carrier transport; surface recombination.

The built-in electric field in the emitter region of $n^{+}$ $p$ structure has the direction opposite to the direction of the minority carrier diffusion flow, and for $p^{+}-n$ structure that coinciding with it. Therefore, independently on the structure type, this field counteracts the diffusion spreading of carriers, the stronger the larger gradient of doping concentration is appeared. Surface recombination at the contact promotes the increase of corresponding component of the diffusion current due to the decrease of the effective lifetime of nonequilibrium carriers.

The relationship (35) can be simplified taking the lifetime of nonequilibrium electrons in the $p$-region at $N_{D}=N_{A}$ equal to the lifetime of nonequilibrium holes in the base. Then (35) takes the form for $p^{+}-n$ structure

$\frac{j_{s n}}{j_{s p}}=\sqrt{\frac{D_{n}}{D_{p}}} e^{-x_{p} / 2 \lambda} \frac{\operatorname{th}\left(d_{n} / L_{p}\right)}{F\left(z_{0}, z_{p}\right)}$.

For $n^{+}-p$ structure the formula (36) takes the form

$\frac{j_{s p}}{j_{s n}}=\sqrt{\frac{D_{p}}{D_{n}}} e^{-x_{n} / 2 \lambda} \frac{\operatorname{th}\left(d_{p} / L_{n}\right)}{F\left(z_{0}, z_{n}\right)}$.

Comparing expressions (32) and (33), as well as (36) and (37), we deduce a conclusion that maximum value of the ratio of the minority carrier current (31) component in the emitter region, that is appreciable because of nonuniform doping profile, to that in the base is reached for $p^{+}-n$ structure $\left(D_{n}>D_{p}\right)$ due to the increase of the base length $\left[\operatorname{th}\left(d / L_{p}\right) \approx 1\right]$ and the impurity concentration $N_{D}$ in the base.

An important thermometric parameter of DTS is the limiting (maximum) temperature $T_{m}$ restricted the effective extent of TRC of a sensor in the range of high tem- 


\section{V.N. Sokolov et al.: Effect of nonuniform doping profile...}

peratures. The temperature $T_{m}$ is defined by the relation $U\left(T_{m}, I\right)=k_{B} T / q[2,3]$. The dependence of $T_{m}$ on the excitation current value $I$, as well as on the design parameters of a diode, can be determined from equation (31) in which the voltage $U$ should be changed by corresponding value of the thermal voltage $k_{B} T_{m} / q$ :

$$
I=(e-1) S_{s}\left(T_{m}, U=k_{B} T_{m} / q\right) .
$$

Moreover, it should be taken into account that the expressions for the saturation current components $j_{s}(T, U)$ are determined by the formulae (32) and (33). Below we present the results of calculations of the temperature $T_{m}$ for the silicon $p^{+}-n$ diode with making use of the following numerical values for the diode structure parameters: $N_{A m}=10^{20} \mathrm{~cm}^{-3}, d_{n}=10 \mu \mathrm{m}, S=410 \mu \mathrm{m}^{2}, \tau_{n}(0)=\tau_{p}=$
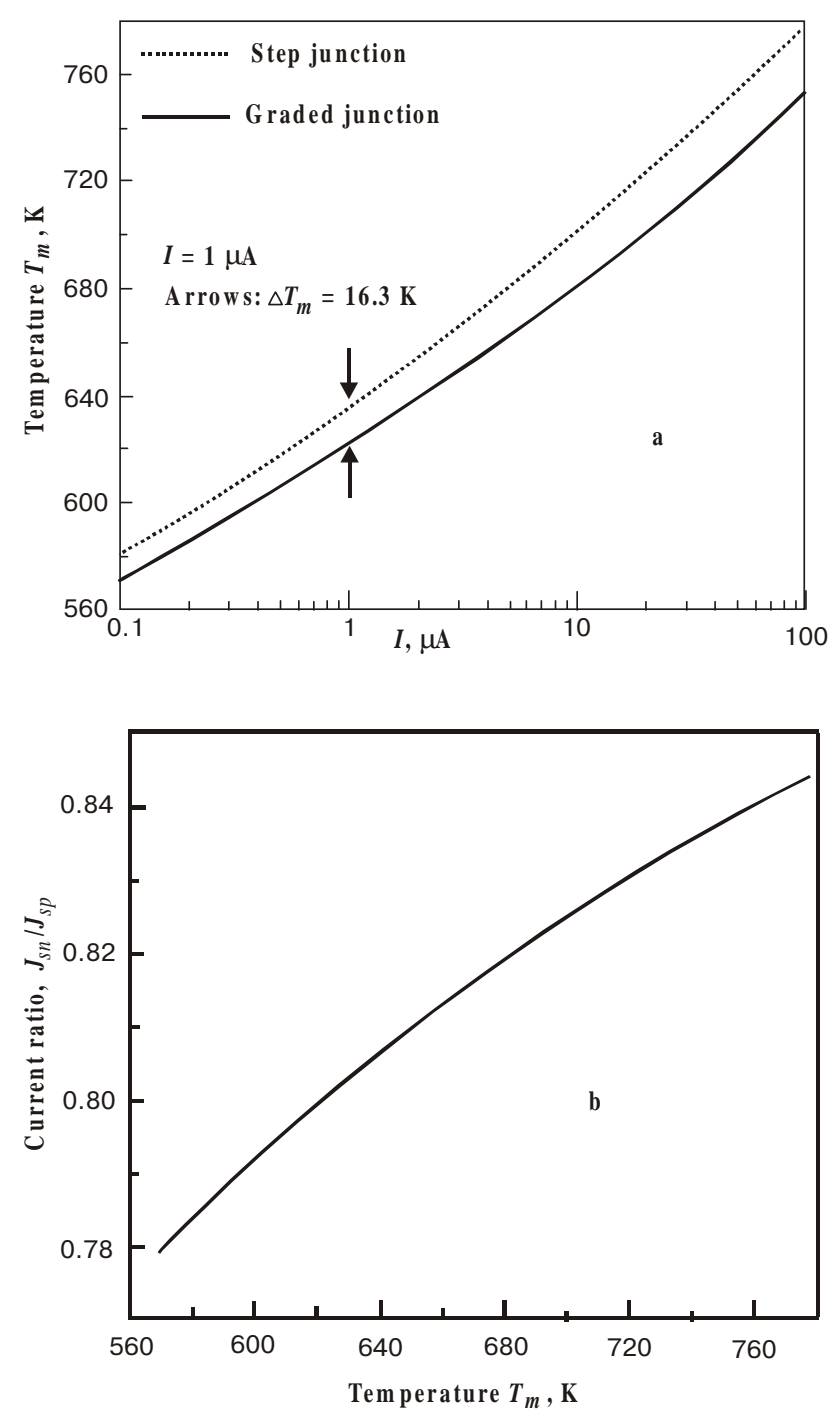

Fig. 2. Dependences of limiting temperature $T_{m}$ on excitation current $I$ (a) and ratio of electron and hole components of the current density $J_{s n} / J_{s p}$ on $T_{m}$ (b) for the $p^{+}-n$ structure with the parameters: $N_{A m}=10^{20} \mathrm{~cm}^{-3}, N_{D}=10^{18} \mathrm{~cm}^{-3}, d_{p}=x_{0}=3 \mu \mathrm{m}$, $d_{n}=10 \mu \mathrm{m}, \quad S=410 \mu \mathrm{m}^{2}$. Dashed curve - stepwise impurity distribution model, solid lines - exponential model.
$=5 \cdot 10^{-9} \mathrm{~s}$. Formulae for calculation of the kinetic coefficients $D_{n, p}$ and $\mu_{n, p}$ were given in [2,3]. The results of calculations are presented in Figs $2-4$. The excitation current value is varied within the range $I=10^{-7} \div 10^{-4} \mathrm{~A}$, the $p$-region length $x_{0}=d_{p}=0.2 \div 10 \mu \mathrm{m}$, the donor concentration in the base $N_{D}=10^{17} \div 10^{18} \mathrm{~cm}^{-3}$.

The dependence of temperature $T_{m}$ on current $I \mathrm{ob}-$ tained by numerical calculations using the formula (38) is presented in Fig. 2 by the solid curve. For a comparison, there is also given analogous dependence for stepwise junction calculated by the formula (3). In Fig. 2, b, we show the dependence of ratio between the electron and hole components of the current $I$ through the $p-n$ junction on $T_{m}$. It is seen that for the considered values of temperature and excitation current of the sensor, the overall current in the $p^{+}-n$ structure is determined by the electron and hole components of the minority carrier current comparable in their magnitudes. As a result, the calculated values of $T_{\mathrm{m}}$ for uniform and exponential impurity distribution in the emitter can distinguish by the value of $\Delta T_{m} \approx 20 \mathrm{~K}$. In particular, for the current $I=1 \mu \mathrm{A}$ we obtain $\Delta T_{m}=16.3 \mathrm{~K}$.

Fig. 3 shows dependences of temperature $T_{m}$ on the $p-n$ junction depth $x_{0}$ calculated for the $p^{+}-n$ structure with donor concentration in the base $N_{D}=10^{18} \mathrm{~cm}^{-3}$ and at the sensor excitation current $I=1 \mu \mathrm{A}$. The dashed line corresponds to stepwise impurity distribution, while the solid curve to the exponential model. It is seen from the calculations that the model of stepwise impurity distribution for asymmetrical $p-n$ junction leads to the temperature $T_{m}$ independent on the $p-n$ junction depth. This is explained by that the minority carrier current component in the emitter region, which, in fact, results in such a dependence on $x_{0}$, is negligible for asymmetrical junction in comparison with the current flowing through $p-n$

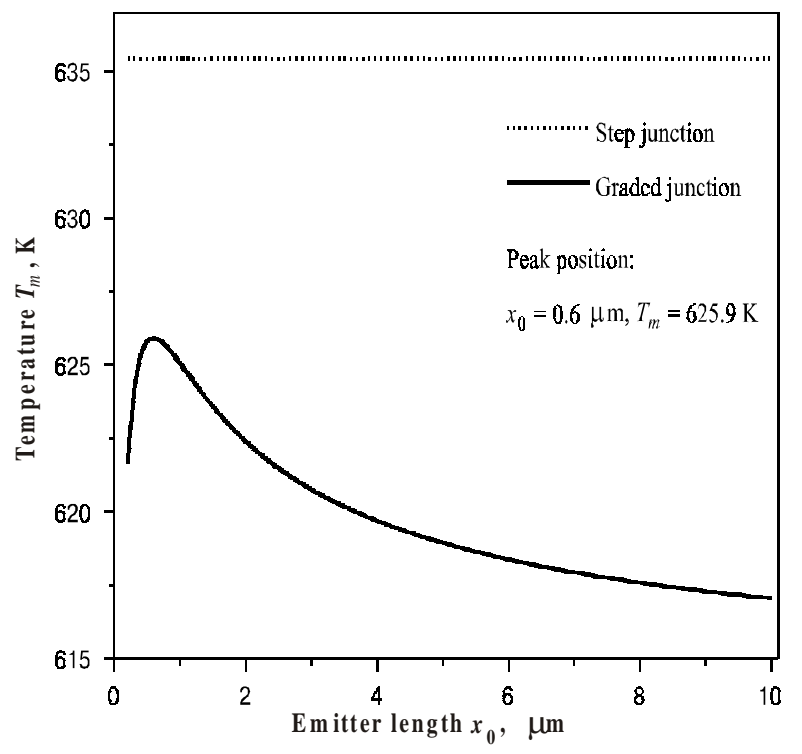

Fig. 3. Dependences of limiting temperature $T_{m}$ on the $p-n$ junction depth $x_{0}: I=1 \mu \mathrm{A}$, the rest parameters are the same as in Fig. 2. Dashed curve - stepwise impurity distribution model, solid lines exponential model. 
junction. In contrast, in the case of nonuniform impurity distribution in the emitter the temperature $T_{m}$ essentially depends on the emitter region length, with a clear maximum being observed in this dependence. Remember that the parameter $x_{0}$ determines the characteristic length scale of impurity distribution (13), the diffusion electric field (14) and, consequently, its magnitude considerably affects the minority carrier transport in this region of the diode. A nonmonotonic behavior of the dependence of $T_{m}$ on $x_{0}$ (solid curve) is due to a concurrence between drift-diffusion and surface recombination flows of nonequilibrium carriers in the emitter region. The maximum value of temperature $T_{m}$ obtained from calculations is $625.9 \mathrm{~K}$ and it isrealized for $x_{0}=0.6 \mu \mathrm{m}$.

In Fig. 4, we show dependences of temperature $T_{m}$ on donor concentration in the base of the $p^{+}-n$ structure calculated for different values of the $p-n$ junction depth $x_{0}$, and for the excitation current value $I=1 \mu \mathrm{A}$. As distinct from the model of stepwise impurity distribution, for which $T_{m}$ does not depend on $x_{0}$ (dashed curve), the calculations (solid curves) demonstrate considerable shift of temperature $T_{m}$ with changing the parameter $x_{0}$ all over the donor concentration range $N_{D}=10^{17} \div 10^{18} \mathrm{~cm}^{-3}$. The results of calculations show that taking account of the nonuniform doping profile in the emitter region of diffused diodes considerably affects TRC of the temperature sensor. At that, such an influence results in not only large in temperature equivalent corrections to theoretical TRC described by the model of stepwise impurity distribution but also, that is especially important, changes qualitatively the behavior of TRC with the change of the diode structure parameters.

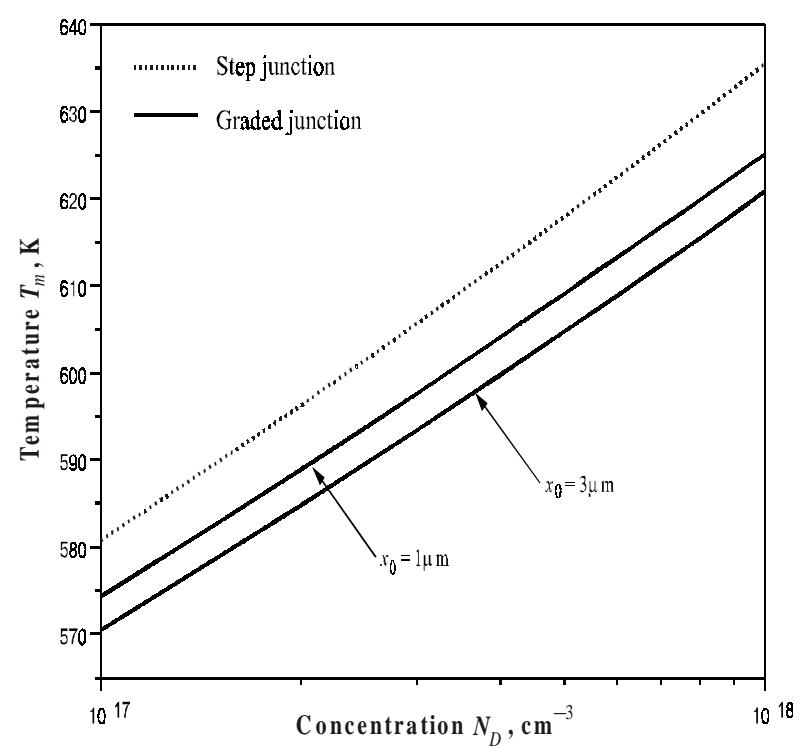

Fig. 4. Dependences of limiting temperature $T_{m}$ on donor concentration $N_{D}$ in the base for different values of the $p-n$ junction depth $x_{0}: I=1 \mu \mathrm{A}$, the rest parameters are the same as in Fig. 2 . Dashed curve - stepwise impurity distribution model, solid lines exponential model.

\section{Conclusions}

In the present paper, a detailed analysis has been carried out of the influence of nonuniform doping profile on the temperature response curve of diode temperature sensor with predominant diffusion carrier transport in silicon structures based on $p-n$ junction. For analytical calculations of CVC and TRC of the diode sensor, the exponentially graded junction model has been used which simplifies integration of Poisson's equation in the heavily-doped emitter region of the diode (with uniform impurity distribution in the base), as well as the depletion layer approximation for the space-charge region of $p-n$ junction.

It has been shown that depending on the values of design parameters of the diode structure, both components of the current through $p-n$ junction, corresponding to the minority carrier transport in the emitter and base, can contribute equivalently to CVC and TRC of the diode sensor. That is, these can determine to the same extent their formation for a specific diode structure. As a result, TRC of the sensor with nonuniform impurity distribution is described by a more complicated temperature dependence of forward voltage at a given value of the current in comparison with that for stepwise impurity distribution model in $p-n$ junction.

A large value of temperature shift of TRC obtained from the calculations (about $20 \mathrm{~K}$ ), resulting from nonuniform doping profile in the emitter region, points to significance of many-factor account of design-technological features of silicon diffused diode structures, widely used for manufacture of temperature sensors.

It is to be noted that there exist several variants of structures distinct on their design used successfully in sensory systems, including those in which the impurity distribution is certainly three-dimensional. The possibility of application of a one-dimensional $p-n$ junction model for calculations of TRC in that case is highly problematic, even with taking into account nonuniform impurity profile in both the emitter and base regions, since a one-dimensional model does not take explicitly into account peculiarities of the carrier transport in the whole structure (field and current density distributions). The calculations of TRC of the sensors based on such structures should be carried out by methods of numerical modeling. For instance, with the aid of numerical simulation of coupled drift-diffusion equations for minority carrier concentrations of electrons and holes as well as the Poisson equation for the electrostatic potential with a real impurity profile. Such calculations, however, are associated with considerable numerical dificulties.

\section{Appendix}

\section{Calculation of space-charge region}

The electrostatic potential and electric field distributions in $p^{+}-n$ structure in the absence of external voltage $(U=0)$ 


\section{V.N. Sokolov et al.: Effect of nonuniform doping profile...}

follow from the solution of Poisson's equation (6) with the boundary conditions corresponding to the field and potential continuity at the space-charge region boundaries. For that, we use the DL approximation, which assumes no free carriers within the DL and the total charge to be due to fully ionized impurities [1]. Bellow, all the quantities with dimensionality of length are normalized to the characteristic scale of impurity distribution $\lambda$ and the electrostatic potential to the thermal voltage $k_{B} T / q$.

As distinct from a stepwise (quasi-uniform) impurity distribution, in the considered case besides the main DL in the vicinity of metallurgical boundary of $p-n$ junction, there is an additional DL [22] at the boundary with uniform $p^{+}$-region of the emitter at $-x_{e} \leq x \leq-x_{0}$, with the extension of $W_{e}=x_{e}-x_{0}$ (Fig. 1). Setting the field $E\left(-x_{e}\right)=0$, and the potential $\varphi\left(-x_{e}\right)=-U_{b i}$, we find for the considered DL the field distribution $E(x)=E_{p r}\left(x+x_{e}\right) / W_{e}$, the potential $\varphi(x)=-U_{b i}+\left[\left(x+x_{e}\right)^{2} /\left(2 W_{e}\right)\right]$, as well as the potential difference $\Delta \varphi_{e} \equiv \varphi\left(-x_{e}\right)-\varphi\left(-x_{0}\right)=-W_{e} / 2$, where $W_{e}=\lambda_{D p}{ }^{2}$ [here the Debye screening length is determined by maximum value of the acceptor concentration in the emitter $\left.\lambda_{D \mathrm{p}}=\left(\varepsilon_{0} k_{B} T / 4 \pi q^{2} N_{A m}\right)^{1 / 2}\right]$.

The quasineutral part of the $p$-region $\left(-x_{0} \leq x \leq-x_{p}\right)$ has the extent $L_{p r}=x_{0}-x_{p}$. For it, characteristic features are linear dependence of the potential $\varphi(x)=\varphi\left(-x_{0}\right)+x+$ $+x_{0}$ and constant field $E(x)=E_{p r}$. The electrostatic potential at the boundary of this part with the main DL of $p-n$ junction is determined by $\varphi\left(-x_{p}\right)=-U_{b i}+\left(W_{e} / 2\right)+L_{p r}$.

The main DL of $p-n$ junction $\left(-x_{p}<x<x_{n}\right)$ has the extent $W=x_{n}+x_{p}$. Taking the field and potential equal to zero in the base of the diode, we find distribution of the field

$$
E(x)=E_{p r}\left(x_{n}-x+e^{-x_{n}}-e^{-x}\right) / \ell_{D n}^{2}
$$

and the potential

$$
\varphi(x)=-\left[\left(x_{n}-x\right)^{2}+2\left(x_{n}-x\right) e^{-x_{n}}+2\left(e^{-x_{n}}-e^{-x}\right)\right] /\left(2 \ell_{D n}^{2}\right)
$$

where $\lambda_{D n}=\left(\varepsilon_{0} k_{B} T / 4 \pi q^{2} N_{D}\right)^{1 / 2}$ is the Debye screening length in the base. Maximum field is localized at the metallurgical boundary $(x=0)$ of $p-n$ junction

$$
E_{m}=E_{p r}\left(x_{n}-1+e^{-x_{n}}\right) / \ell_{D n}^{2} .
$$

The corresponding potential difference $\Delta \varphi_{p n}=$ $=\varphi\left(-x_{p}\right)-\varphi\left(x_{n}\right)=\varphi\left(-x_{p}\right)$ for the main DL is equal to

$$
\varphi_{n p}=-\left[W^{2}+2 W e^{-x_{n}}+2\left(e^{-x_{n}}+e^{x_{p}}\right)\right] /\left(2 \ell_{D n}^{2}\right)
$$

The built-in potential $U_{b i}$ is determined by the condition of the Fermi level constancy in the $p^{+}-n$ structure $U_{b i}=\ln \left(N_{A m} N_{D} / n_{i}^{2}\right)[1]$.

The extent of the region of positive $\left(x_{n}\right)$ and negative $\left(x_{p}\right)$ charge can be obtained from the system of transcendental equations, which express the condition of field and potential continuity on the DL boundary at $x=-x_{p}$. Tak- ing in accordance with the accepted model (see Introduction) the applied voltage to drop on the main DL of $p-n$ junction, we can write these equations as follows

$$
\begin{aligned}
& W-x_{n}-x_{p}=0 \\
& W+e^{-x_{n}}-e^{x_{p}}=\ell_{D n}^{2}, \\
& W^{2}+2 W e^{-x_{n}}+2\left(e^{-x_{n}}-e^{x_{p}}\right)+2 l_{D n}^{2}\left(x_{0}-x_{p}\right)= \\
& =2 \ell_{D n}^{2}\left(U_{b i}-U\right) .
\end{aligned}
$$

The solution of (П5) for $U>0$ gives the dependence of the DL boundaries, $x_{n}(U)$ and $x_{p}(U)$, on the applied voltage $U$ for a forward biased diode. For calculation of CVC of the diode and TRC of the temperature sensor, these dependences should be substituted into formulae (31) - (33) and (35)-(38), respectively.

\section{References}

1. S.M. Sze, Physics of Semiconductor Devices, $2^{\text {nd }}$ ed. (Wiley, New York, 1981).

2. Yu.M. Shwarts, V.L. Borblik, N.R. Kulish, E.F. Venger, V.N. Sokolov, Limiting characteristics of diode temperature sensors // Sensors and Actuators A: Physical, 86 (3), pp.197$205(2000)$

3. N.R. Kulish, Yu.M. Shwarts, V.L. Borblik, E.F. Venger, V.N. Sokolov, Self-consistent method for optimization of parameters of diode temperature sensors // Semicond. Phys., Quant. Electron. \& Optoelectron., 2 (2), pp.15-27 (2000).

4. J.K. Krause, B.C. Dodrill, Measurement system induced errors in diode thermometry // Rev. Sci. Instr., 57 (4), pp.661665 (1986).

5. P. Ashburn, D.V. Morgan and M.J. Howes, A theoretical and experimental study of recombination in silicon $p-n$ junctions // Solid-State Electron., 18 (6), pp.569-577 (1975).

6. G.F. Cerofolini and M.L. Polignano, Generation-recombination phenomena in almost ideal silicon $p-n$ junctions // J. Appl. Phys. 64 (11), pp.6349-6355 (1988).

7. G.F. Cerofolini and M.L. Polignano, Residual non-idealities in the almost ideal silicon $p-n$ junction // Appl. Phys. A 50 (2), pp.273-286 (1990).

8. J.A. Del Alamo and R.M. Swanson, Forward-bias tunneling: A limitation to bipolar device scaling // IEEE Electron Device Lett. EDL-7 (11), pp.629-631 (1986).

9. G.I. Andersson and O.Engström, Forward-bias tunneling at defect clusters in silicon emitter junctions // J. Appl. Phys. 69 (8), pp.4418-4425 (1991).

10. J.C.S. Woo, J.D. Plummer, and J.M.C. Stork, Non-ideal base current in bipolar transistors at low temperatures // IEEE Trans. Electron Devices ED-34 (1), pp.130-138 (1987).

11. E. Simoen, J. Vanhellemont, A.L.P. Rotondaro and C. Clayes, Static and low-frequency noise characteristics of $n^{+} p$ junction diodes fabricated in different silicon substrates // Semicond. Sci. Technol. 10 (7), pp.1002-1008 (1995).

12. Yu.M. Shwarts, V.N. Sokolov, M.M. Shwarts, I.A. Fyodorov, E.F. Venger, Silicon diode temperature sensors for cryogenic application // The MTEC Intern. Conf. on Sensors \& Transducers. NEC.- Birmingham (UK). $-2000 .-$ pp.1-10.

13. D. Redfield, Revised model of asymmetric $p-n$ junctions // Appl. Phys. Lett. 35 (2), pp.182-184 (1979).

14. J.S. Hamel, Simplified analytical quasi-transparent solution for minority carrier transport in non-uniformly doped quasineutral semiconductor regions // IEEE Trans. Electron Devices 43 (1), pp.104-109 (1996). 
V.N. Sokolov et al.: Effect of nonuniform doping profile...

15. B.S. Wu and F.A. Lindholm, One-dimensional all injection nonquasi-static models for arbitrarily doped quasi-neutral layers in bipolar junction transistors including plasma-induced energy-gap narrowing // IEEE Trans. Electron Devices 37 (1), pp.250-261 (1990).

16. L.A. Vernoef and W.C. Sinke, Minority-carrier transport in nonuniformly doped silicon - an analytical approach // IEEE Trans. Electron Devices 37 (1), pp.210-221 (1990).

17. B.G. Cohen, W.B. Snow and A.R.Tretola, GaAs $p-n$ junction diodes for wide range thermometry // Rev. Sci. Instr., 34 (10), pp.1091-1093 (1963).

18. N. Sclar and D.B. Pollock, On diode thermometers // SolidState Electron. 15 (5), pp.473-480 (1972).

19. V.K. Aladinskiy, V.F. Baryshnikov, V.G. Solyar, Temperature dependence nonlinearity calculation of forward voltage of $p-n$ junction // Elekronnaya tehnika, Ser. 2 (4), pp.35 (1986).

20. V.I. Stafeev, Effect of the resistance of the bulk of a semiconductor on the form of the current-voltage characteristic of a diode // ZhTF 28 (8), p.1631-1641 (1958) [Soviet physics technical physics 3(8), 1502-1512 (1958) ].

21. Yu.M. Shwarts, V.L. Borblik, N.R. Kulish, V.N. Sokolov, M.M Shwarts, E.F. Venger, Silicon diode temperature sensor without a kink of the response curve in cryogenic temperature region // Sensors and Actuators A: Physical, 76 (1-3), pp.107111 (1999).

22. J.M. Pimbley, Depletion approximation analysis of an exponentially graded semiconductor $p-n$ junction // IEEE Trans. Electron Devices ED-35 (11), pp.1957-1962 (1988).

23. S.E. Schacham and E. Finkman, Saturation current and excess carrier distribution in exponentially graded $p-n$ junctions // J. Appl. Phys. 71 (10), pp.5033-5040 (1992).

24. N. Rinaldi, Analysis of the deplition layer of exponentially graded $p-n$ junctions with nonuniformly doped substrates // IEEE Trans. Electron Devices 47 (12), pp.2340-2346 (2000).

25. D.S. Kuznetsov, Special functions. Vysshaya shkola, Moscow, 1965, p.121. 